\title{
Malaria Theranostics using Hemozoin-Generated Vapor Nanobubbles
}

\author{
Ekaterina Y. Lukianova- Hleb ${ }^{1}$, Dmitri O. Lapotko ${ }^{1,2}$ \\ 1. Department of Biochemistry and Cell Biology, Rice University, Houston, TX; \\ 2. Department of Physics and Astronomy, Rice University, Houston, TX.
}

$\bowtie$ Corresponding author: d15@rice.edu.

( ) Ivyspring International Publisher. This is an open-access article distributed under the terms of the Creative Commons License (http://creativecommons.org/ licenses/by-nc-nd/3.0/). Reproduction is permitted for personal, noncommercial use, provided that the article is in whole, unmodified, and properly cited.

Received: 2014.03.16; Accepted: 2014.04.26; Published: 2014.05.22

\begin{abstract}
Malaria remains a widespread and deadly infectious human disease, with increasing diagnostic and therapeutic challenges due to the drug resistance and aggressiveness of malaria infection. Early detection and innovative approaches for parasite destruction are needed. The high optical absorbance and nano-size of hemozoin crystals have been exploited to detect and mechanically destroy the malaria parasite in a single theranostic procedure. Transient vapor nanobubbles are generated around hemozoin crystals in malaria parasites in infected erythrocytes in response to a single short laser pulse. Optical scattering signals of the nanobubble report the presence of the malaria parasite. The mechanical impact of the same nanobubble physically destroys the parasite in nanoseconds in a drug-free manner. Laser-induced nanobubble treatment of human blood in vitro results in destruction of up to $95 \%$ of parasites after a single procedure, and delivers an 8 -fold better parasiticidal efficacy compared to standard chloroquine drug treatment. The mechanism of destruction is highly selective for malaria infected red cells and does not harm neighboring, uninfected erythrocytes. Thus, laser pulse-induced vapor nanobubble generation around hemozoin supports both rapid and highly specific detection and destruction of malaria parasites in one theranostic procedure.
\end{abstract}

Key words: malaria, hemozoin, laser, vapor nanobubble, theranostics.

\section{Introduction}

Innovative methods are required to combat growing drug resistance of malaria parasites [1-9]. The detection of parasites and their destruction require sensitivity and specificity for single infected cells, using robust, reliable, and inexpensive devices with a minimal dependence upon chemical reagents. None of the existing technologies can rapidly and non-invasively detect and destroy the parasite with single red blood cell sensitivity, and this problem is exacerbated by the evolution of drug-resistant strains of malaria parasites.

The blood-stage malaria parasite uses hemoglobin as a nutrient source, releasing large amounts of potentially toxic heme, which generates reactive ox- ygen species, leading to parasite death. To circumvent this internal toxicity, the parasite packages the heme groups into unique and biochemically inert nanocrystals called hemozoin particles that are located in food vacuoles inside a parasite [10-15]. We have previously shown that the high optical absorbance of the hemozoin nanocrystal can be used to generate transient localized vapor nanobubble in response to a short laser pulse [16]. The optical absorbance of hemozoin significantly exceeds that of any of the individual heme containing proteins found uninfected blood cells and tissue (i.e. hemoglobin, myoglobin, cytochromes, etc.). The hemozoin nanocrystal converts the optical energy of a short laser pulse into the 
localized heat which rapidly generates a localized transient vapor nanobubble around the hemozoin nanocrystal in the malaria parasite within an infected red blood cell (iRBC) (Figure 1A). Unlike many current malaria treatments that combat the parasite by inhibiting hemozoin formation and allowing heme toxicity [3], we propose to use hemozoin nanocrystals as an Achilles heel to both detect its presence via the optical scattering by a vapor nanobubble (Figure 1B) and destroy the parasite mechanically with the intra-parasital explosive impact of vapor nanobubble (Figure 1C), all in one theranostic procedure. In our recent study, we demonstrated the diagnostic proper- ties of hemozoin-generated vapor nanobubbles (H-VNB) using transdermal acoustic detection [16] and suggested that this technology might also be used to selectively destroy malaria parasites in infected red blood cells without damaging neighboring uninfected cells in whole blood (Figure 1C). In this report, we have now examined in detail the parasiticidal capabilities of $\mathrm{H}-\mathrm{VNB}$ in Plasmodium falciparum-infected human red blood cells, which, in combination with our previous study on diagnosis, demonstrates unambiguously the theranostic capabilities of this technology.
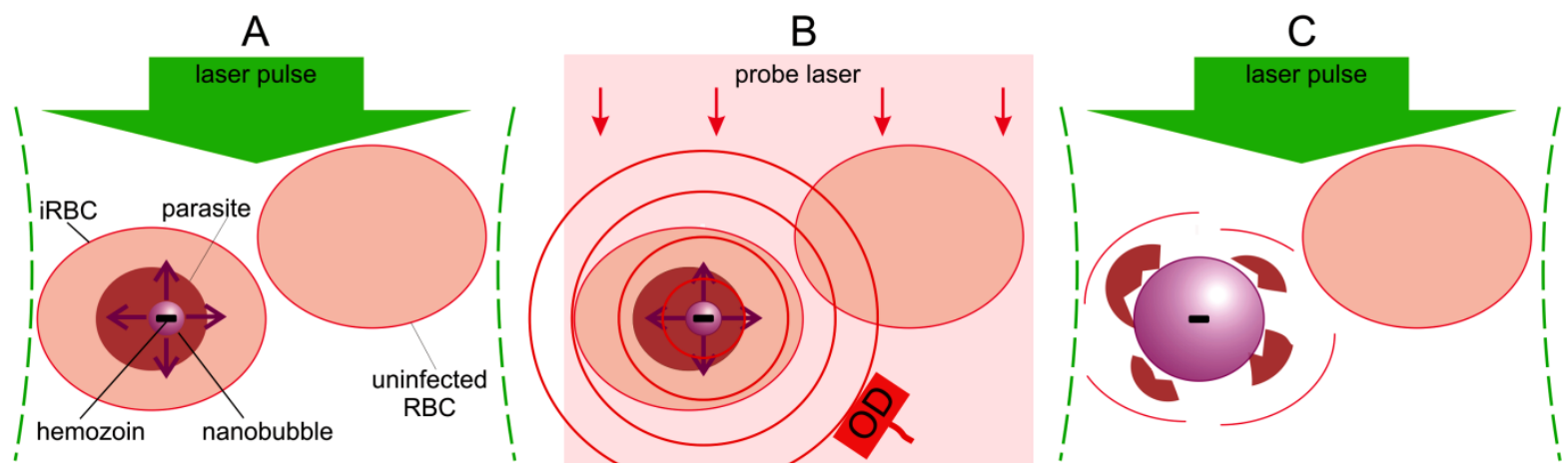

Figure I. Principle of malaria theranostics with hemozoin-generated vapor nanobubbles (H-VNB). (A): Selective generation of a transient $\mathrm{H}-\mathrm{VNB}$ around hemozoin nanocrystal inside a malaria parasite within an infected red blood cell (iRBC). (B): Detection of optical scattering signals from the H-VNB can occur with an additional probe laser (red) and optical detector (OD). (C): The mechanical destruction of the parasite occurs by explosive expansion and collapse of the H-VNB during its nanosecond lifespan. There would be no effect on nearby uninfected red blood cells, which lack hemozoin particles.

\section{Materials and Methods}

\section{Generation and detection of vapor nanobubbles around hemozoin nanocrystals}

Generation of the expanding and collapsing vapor nanobubble assumes transient localized evaporation of the liquid around hemozoin nanocrystal. This vaporization is achieved through the photothermal effect in hemozoin nanocrystal. Optical energy is absorbed by the nanocrystal, instantaneously converted into heat by hemozoin, and then this heat is transferred to an adjacent liquid, which is rapidly evaporated. This process results in an expanding and collapsing vapor nanobubble [16-18]. The duration of the expansion-collapse cycle determines the lifetime of a nanobubble (from $10 \mathrm{~ns}$ to microseconds), which is proportional to the maximal diameter of the optically absorbing object $[17,18]$ and is used as the main metric of the nanobubble. The energy efficacy of H-VNB generation depends upon the nanocrystal size and the laser pulse duration. The latter has to be much shorter than the characteristic cooling time of the laser-heated, optically absorbing object (hemozoin nanocrystal in our case) [19]. The sizes of hemozoin nanocrystals vary in the range $50-1000 \mathrm{~nm}$, with the smallest nanocrystals being associated with the early ring stage of the malaria parasite [13-15]. This size range corresponds to cooling times in the range 0.5 26 ns. To ensure efficient thermal confinement and thus to maximize the energy efficacy of the $\mathrm{H}-\mathrm{VNB}$ generation, we used 70 ps laser pulses and compared their efficacy to a longer, 14 ns pulses, that allow more diffusive heat dissipation. The duration of the $70 \mathrm{ps}$ pulse is much shorter than the above estimates for cooling times, and therefore such pulse durations should provide very localized heating with minimal heat losses during the generation of H-VNBs. The excitation wavelength was chosen to be $532 \mathrm{~nm}$ where hemozoin shows relatively high optical absorbance [11]. The 70 ps pulse was delivered by a mode locket Nd-YAG laser (PL-2250, Ekspla, Vilnius, Lithuania) and the longer $14 \mathrm{~ns}$ pulse by another Nd-YAG laser (LS-2145T, Lotis TII, Minsk, Belarus). The optical microscope-based experimental set up has been described in detail previously [16, 17, 20-22].

In the experiments shown in Figure 2, H-VNBs generated in response to a single pulse were detected with two optical methods: (1) nanobubble-specific optical scattering responses of a continuous probe 
laser beam [16], and (2) optical scattering time-resolved images which were been obtained with the delayed probe laser pulse [16, 17]. Both signals were recorded simultaneously during exposure of each cell to a single laser pulse. The nanobubble lifetime (duration of the light scattering response in Figure 2D) was used as a metric for H-VNB size, its diagnostic sensitivity, and parasiticidal efficacy.

Studies of the individual cells included several steps. A cell was placed into the center of laser beam, and a bright field image of the cell was obtained to identify the cell (Figure 2A). A SYBR green I fluorescent image was obtained to identify the malaria para- site by DNA staining (Figure 2B). A single laser pulse was applied at specific fluence, and H-VNB optical signals were simultaneously recorded by using the excitation laser pulse to trigger the optical detector and the oscilloscope to record the optical scattering image of $\mathrm{H}-\mathrm{VNB}$ (Figure 2C) and the time-response of $\mathrm{H}-\mathrm{VNB}$ (Figure 2D). Ten seconds after the laser pulse, the second bright field image of the cell was obtained to look for cellular damage (Figure 2E). For each irradiated cell, the location of the parasite (fluorescent image) was correlated to the location of the H-VNB image.
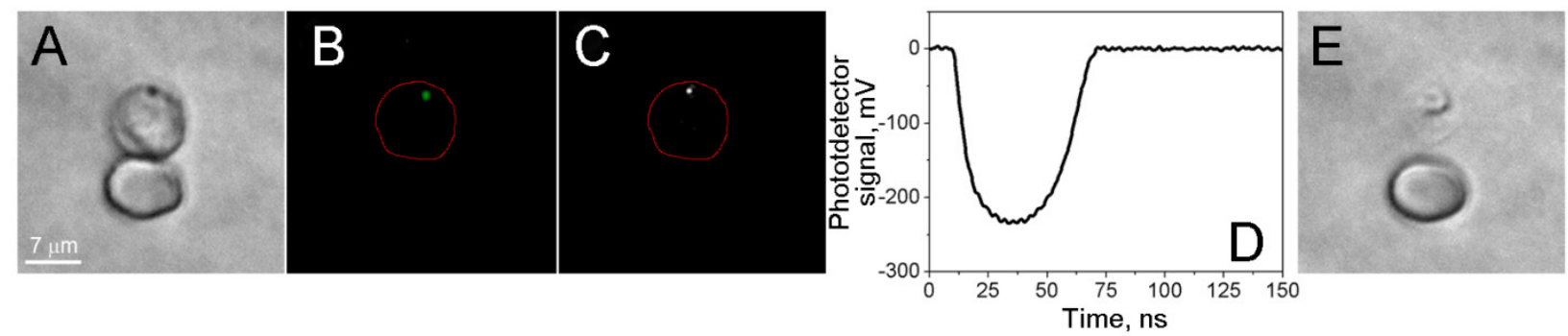

Figure 2. Localized disruptive effect of laser-induced nanobubbles. Images of two RBCs, parasite-infected (top) and uninfected (bottom) human red blood cells. (A) Bright field image before the nanobubble generation. (B) Fluorescent image of SYBR green I before the nanobubble generation. (C) Time-resolved optical scattering image of a nanobubble under a short pulse laser excitation. (D) Optical scattering trace with nanobubble-specific signal. (E) Bright field image after nanobubble generation shows the destroyed host cell and the altered image of the parasite.

\section{Hemozoin crystals}

Isolated hemozoin nanocrystals in water have been used to verify the H-VNB generation and detection methodology. The hemozoin crystals $(5 \mathrm{mg}$, InvivoGen, \#HMZ-33-04) were diluted by $1 \mathrm{ml}$ of sterile phosphate buffered saline ( $\mathrm{pH} 7.4)$. This suspension was mixed and sonicated ( $5 \mathrm{~min}$, room temperature) to obtain a homogenous dispersion of the crystals. After that the stock suspension was diluted in 1000-fold for studying the individual hemozoin crystals and $5 \mu \mathrm{l}$ of this working suspension was dispersed on standard microscope slides and coverslips. The optical scattering images of the individual crystals were used for positioning them into the center of laser beams [16].

\section{Malaria model}

P. falciparum, strain 3D7, was obtained from red blood cell stabilates preserved in liquid nitrogen (the level of parasitemia during storage is $\geq 10 \%$ ) (MRA-1001, ATCC Manassas Virginia). Asynchronous cultures (with the ring and schizont type present) were maintained on plates at $37^{\circ} \mathrm{C}$ at $5 \%$ parasitemia in RPMI 1640 (\#31800-022, Gibco-Life Technology, Rockville, MD) supplemented with $0.5 \%$ Albumax II (\#11021-029, Gibco-Life Technology, Rockville, $\mathrm{MD}$ ) under a $5 \% \mathrm{O}_{2} / 5 \% \mathrm{CO}_{2} / 90 \% \mathrm{~N}_{2}$ atmos- phere as previously described by Trager and Jensen [23].

Two standard microscopy methods were used to detect and quantify the malaria infections. Giemsa staining was used as a standard approach to identify ring and schizont stages of malaria parasite development [24] and to measure the level of parasitemia, defined as the ratio of the malaria parasite-infected cells to the total number of cells. In addition, we stained the cells with SYBR green I (\#S7563, Molecular Probes, Eugene, OR) and applied fluorescent imaging to identify malaria parasites in cells $[12,16,25,26]$. For fluorescent imaging of the parasites, a solution of SYBR green I was added to an aliquot of a stock culture, the suspension was mixed, and the sample placed in the dark for $5 \mathrm{~min}$. Cells were washed twice with complete medium to remove unbound SYBR green I before imaging. These two staining methods were applied in combination with laser scanning confocal microscopy (LSM 710, Carl Zeiss Inc.). This combination of techniques ensured high quality of the bright field and fluorescent imaging. From 10 to 20 image frames were collected to analyze the images of 2,500-5,000 cells to identify and count infected cells and to identify the malaria parasite development stage. We observed good correlation between the SYBR green I- and Giemsa-based methods [16]. Both staining techniques were also used for analyzing the 
percentage of infected cells before and after the treatment of the blood samples with H-VNBs or the anti-malaria drug chloroquine (C6628, Sigma-Aldrich LLC, Saint Louis, MO). For the experiments with individual cells, RBC suspensions $\left(7 \times 10^{5}\right.$ cells $\left./ \mathrm{ml}\right)$ were placed on Ibidi 6 -channel plates ( $\mu$-Slide VI 0.4, \#80606, Ibidi, LLC., Verona, WI). Experiments were repeated three times under identical conditions.

\section{Flow treatment of the infected blood}

We designed a closed sterile flow system (Figure 3 ) that included an optically transparent flow cuvette ( $\mu$-Slide VI 0.1, \#80666, Ibidi, LLC., Verona, WI) connected to two syringes, one dispensing and one collecting the red blood cell suspension. Both syringes were synchronously driven with computer-controlled pumps (NE-1000, New Era Pump Systems, Inc.,
Farmingdale, NY). The excitation laser beam was directed through the cuvette. The geometry of the channel (rectangular cross-section $1 \mathrm{~mm}$ wide, $0.1 \mathrm{~mm}$ deep and $15 \mathrm{~mm}$ length) ensures laminar flow with a two-dimensional monolayer of flowing cells being formed in the middle of the cuvette. The syringes were kept at physiological temperature by automated heating sleeves. The diameter of the excitation laser beam was increased to $1.8 \mathrm{~mm}$ to provide uniform irradiation of all cells in the $1 \times 1 \mathrm{~mm}$ area of the cuvette for each pulse. Flow rate was adjusted to the laser pulse repetition rate $(10-40 \mathrm{~Hz})$ to ensure single pulse exposure of each cell flowing through the $\mathrm{cu}-$ vette. A low flow rate was used to treat $1 \mathrm{ml}$ of the cell suspension in several minutes.
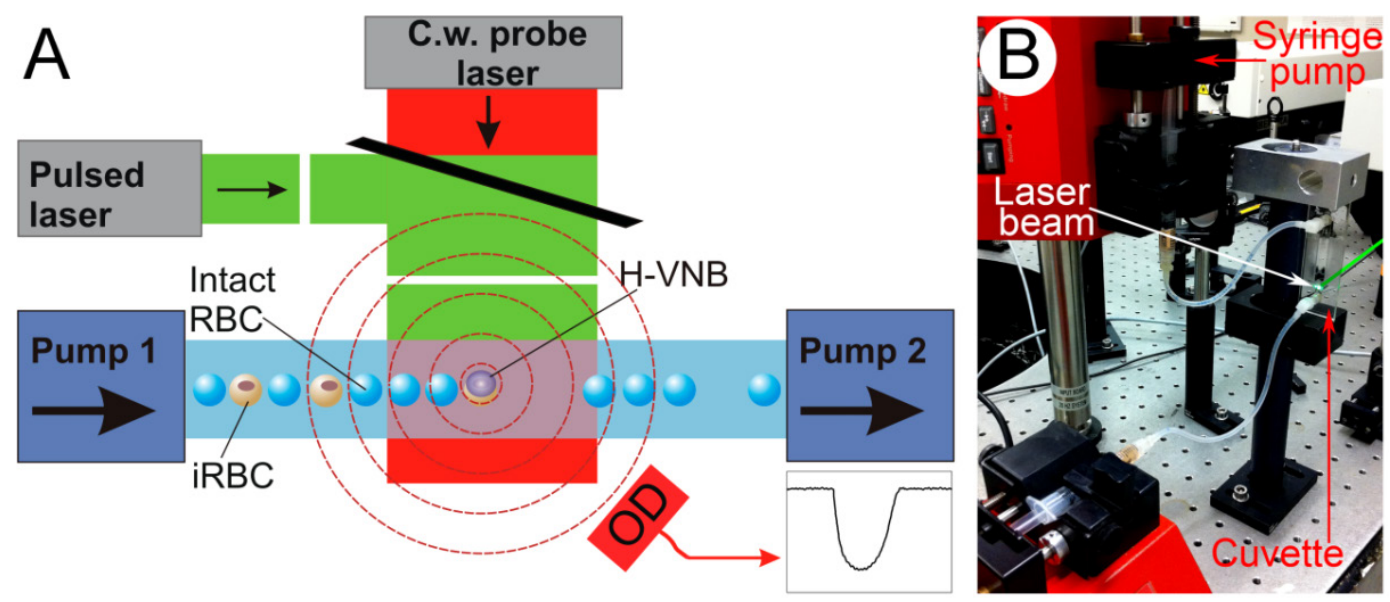

Figure 3. Experimental setup: (A) Scheme for the bulk flow treatment of blood cells with a pulsed broad excitation laser and a flow cuvette with two pumps for dispersing and collecting of blood cells. Continuous low power probe laser beam (red) is side-scattered by $\mathrm{H}$-VNBs and its scattered radiation is registered by an optical detector. (B) Experimental prototype.

We applied the following protocol for the flow treatment of the malaria parasite-infected red cells. The initial level of parasitemia was calculated with the two staining methods described above, and the cell suspension was adjusted to $3 \times 10^{6} \mathrm{cell} / \mathrm{ml}$. Cells were pumped through the system and then exposed to a specific pulsed laser fluence. Cells were collected from the exit of the flow cuvette and then cultured for $48 \mathrm{~h}$. Cell concentration and the levels of parasitemia were measured before and 24 and $48 \mathrm{~h}$ after treatment.

In the experiments with drug treatment, chloroquine (C6628, Sigma-Aldrich LLC, Saint Louis, MO) was added to the cell suspensions immediately prior to the flow treatment. A drug dose of $1 \mu \mathrm{M}$ was calculated to match the therapeutic level used in most treatment regimens [12]. Each treatment was repeated 3-4 times for different blood samples, each of which was cultured independently.

The parasiticidal effect of the bulk flow treat- ment was analyzed using three parameters. (1)The absolute number of malaria parasite-infected cells (parasitemia level) for the 24- and 48-hour time points was measured and compared to that of the initial untreated samples. This metric was used to estimate the efficacy of the specific treatment mode and to compare different treatments at one time-point. (2) The relative level of malaria parasite-infected cells was calculated for each time point as the ratio of the absolute number of malaria parasite-infected cells in the treated sample to that in the untreated control with intact blood cells. This metric was used to compare the parasiticidal kinetics in the different non-synchronyzed samples of parasite-infected blood cells. (3) The total red cell concentration characterized the safety of the treatment for uninfected cells. The concentration of red blood cells was measured at each time point and compared to the initial concentration of the cells in the suspension prior to flow treatment. 


\section{Results}

\section{Generation and detection of the H-VNB in malaria-infected human red blood cells}

We cultured malaria parasites, P. falciparum (strain 3D7), in human blood and exposed individual iRBCs to single laser pulses (70 ps or $14 \mathrm{~ns}, 532 \mathrm{~nm}$ ). The presence and stage of the parasite in each cell were verified with the two independent staining methods, Giemsa treatment with bright field imaging (Figure 2A) and SYBR green I staining with fluorescence imaging (Figure 2B). The generation of the H-VNBs was detected by time-resolved optical scattering imaging (Figure 2C) and time courses (Figure 2D). Both signals showed the rapid appearance and decay of transient vapor nanobubbles with lifetimes on nanosecond scales. A bright flash is seen in the scattering image (Figure 2C), and the expansion and collapse of the H-VNB is reported in the optical scattering time-response (Figure 2D). The position of the H-VNB coincides with that based on staining of the malaria parasite in the same iRBC (Figure 2A\&B) This control experiment demonstrates that the generation of H-VNBs occurs specifically in the P.falciparum parasite and not in adjacent uninfected cells (Figure 2). The latter show no optical scattering signals in response to the laser pulse, indicating the specificity of the method for only infected red blood cells.

\section{Destruction of malaria parasites with the H-VNBs}

The generation of a vapor nanobubble around hemozoin particles results in the destruction of the parasite and the infected red cell after a single laser pulse (Figure 2E). In contrast, irradiation of uninfected RBCs under the same conditions does not generate any vapor nanobubbles as detected by light scattering images and time responses. Even more importantly, no signs of laser-induced damage or significant heating of the uninfected RBCs are observed. The selective generation of $\mathrm{H}-\mathrm{VNBs}$ in only iRBC results from the combination of: (1) the 5- to 7-fold higher optical absorbance of hemozoin compared to that of the hemoglobin solution in the RBC cytoplasm [11]; (2) the temporally and spatially localized heat release and evaporation of liquid inside the parasite due to the nano-size of the hemozoin nanocrystals located inside the parasite; and (3) the short duration of the laser pulse (70 ps), which prevents thermal losses from nanocrystal due to thermal diffusion $[17,19]$.

Rapid expansion and collapse of the H-VNB takes tens and hundreds of nanoseconds and is co-localized with hemozoin, which is found in the parasite food vacuole [10-15]. The expansion and contraction of the H-VNB occurs inside the parasite and mechanically destroys it with nanosecond explosion. Although the parasite and infected red cell are broken apart, the H-VNB seems not to immediately destroy the exposed DNA, which still binds the SYBR green I fluorescence dye, even after disruption of the parasite and red cell membranes (Figure 4). DNA fluorescence (Figure 4B) is detectable for several hours in the cell fragments after $\mathrm{H}-\mathrm{VNB}$ generation and the parasite destruction (Figure $4 \mathrm{C} \& D$ ). In order to directly prove parasite death by the H-VNB, we monitored the parasite activity and level of parasitemia in blood after bulk H-VNB treatment.

The malaria parasite activity was analyzed through the level of parasitemia before and after the single bulk flow laser treatment of human blood infected with P.falciparum (Figure 3). We applied $70 \mathrm{ps}$ pulses at the fluence level of $30 \mathrm{~mJ} / \mathrm{cm}^{2}$ that corresponds to 40-60 ns lifetimes of the H-VNBs in iRBCs. The flow rate, laser beam diameter and laser pulse repetition rate were synchronized to provide a single laser pulse exposure to each cell flowing through the system. The level of parasitemia and the cellular concentrations were measured for 3000-4000 cells at three time-points: 0 (before treatment) and 24 and 48 hours after treatment, using the Giemsa bright field method (Figure 5). These time intervals are long enough to allow significant multiplication of any viable parasites that might have survived H-VNB treatment. For the untreated samples of malaria-infected cells, these time interval demonstrated significant growth of the malaria parasites: the level of parasitemia has increased by one order of magnitude (Figure 5 , grey).

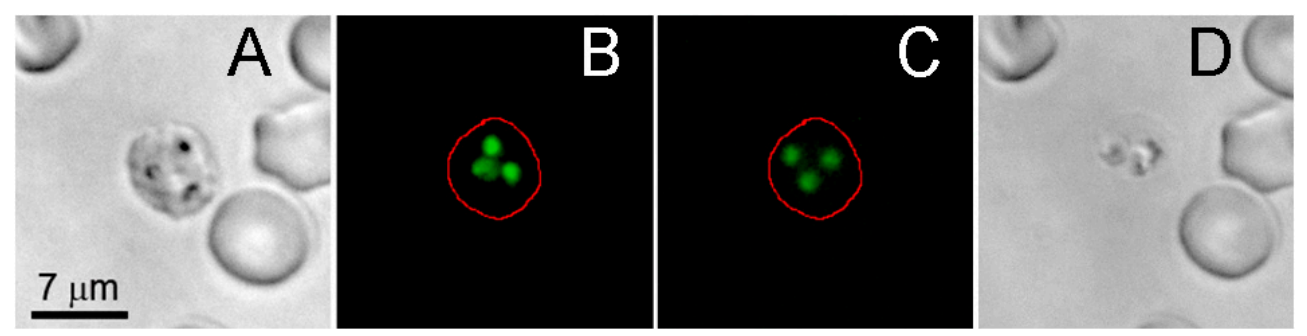

Figure 4. Influence of the laser-induced nanobubble on the location and integrity of SYBR green I-stained malaria parasite in schizont stage of an individual infected red blood cell. (A) The bright field image of the cell before the laser pulse. (B) SYBR green I fluorescence of the cell before the laser pulse. (C) SYBR green I fluorescence of the same cell $2.5 \mathrm{~h}$ after nanobubble generation. (D) The bright field image of the same cell $2.5 \mathrm{~h}$ after nanobubble generation. 

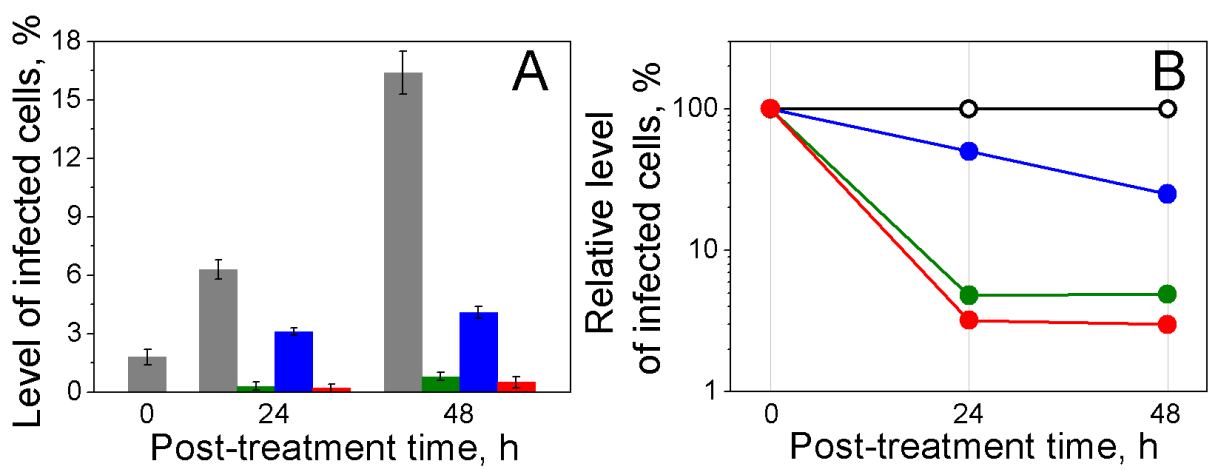

Figure 5. Parasiticidal effects on $P$ falciparum-infected human RBCs exposed to laser pulse in bulk culture. (A): Levels of infected cells: initially $(0 \mathrm{~h})$ and 24 $\mathrm{h}$ and $48 \mathrm{~h}$ after laser flow treatment (grey: untreated iRBCs; green: iRBCs treated with laser pulse of $70 \mathrm{ps}, 30 \mathrm{~mJ} / \mathrm{cm}^{2} ;$ blue: iRBCs treated with I $\mu M$ chloroquine; red: iRBCs treated with laser pulse of $70 \mathrm{ps}, 30 \mathrm{~mJ} / \mathrm{cm}^{2}$ and I $\mu \mathrm{M}$ chloroquine). (B) Relative levels of infected cells 0,24 and $48 \mathrm{~h}$ after laser flow treatment: (black: untreated iRBCs; green: iRBCs treated with laser pulse of $70 \mathrm{ps}, 30 \mathrm{~mJ} / \mathrm{cm}^{2}$; blue: iRBCs treated with I $\mu M$ chloroquine; red: iRBCs treated with laser pulse of $70 \mathrm{ps}, 30 \mathrm{~mJ} / \mathrm{cm}^{2}$ and I $\mu \mathrm{M}$ chloroquine).

After a single bulk H-VNB treatment, the observed lack of multiplication of parasites and, more importantly, the decrease in the level of parasitemia after the treatment (Figure 5, green) are almost certainly due to H-VNB-induced destruction of the parasites. Generation of H-VNBs in the malaria parasite also often induced lysis of the host cells (Figure 2E) due to mechanical perforation of red cell membranes. It was difficult to count the parasite destruction and the lysis of the iRBCs (via the cell concentration) directly due to the low initial level of iRBCs. Nevertheless, potential escape of the viable parasites from disrupted iRBCs was not observed because the level of the parasitemia decreased markedly in the treated suspension even after 24 and $48 \mathrm{~h}$ incubations. In contrast, any viable parasites that escaped iRBCs would have inevitable caused a radical increase in the level of parasitemia, which did not happen.

The destructive effect of $\mathrm{H}-\mathrm{VNB}$ was completely malaria parasite-specific because uninfected red blood cells showed no detectable damage (Figure 2E) and the concentration of uninfected RBCs did not show any detectable changes 24 or $48 \mathrm{~h}$ after the $\mathrm{H}-\mathrm{VNB}$ treatment and was $3.0 \times 10^{6}$ cells $/ \mathrm{ml}$ and $2.95 \times 10^{6}$ cells $/ \mathrm{ml}$, respectively (compared to the initial cell concentration of was $3.0 \times 10^{6}$ cells $\left./ \mathrm{ml}\right)$. This result confirms the localized, malaria parasite-specific nature of the parasiticidal effect of H-VNBs whose lethal mechanical impact was confined by inactivation of their multiplication in culture compared to the untreated sample. This experiment demonstrates that $\mathrm{H}-\mathrm{VNB}$ acts as a parasite-specific intracellular parasiticidal agent which selectively destroys a parasite.

\section{Parasiticidal effects of the H-VNBs vs standard anti-malaria drugs}

In the second sample, in addition to the bulk laser treatment, we applied the standard malaria drug chloroquine in a therapeutic dose of $1 \mu \mathrm{M}$ [26]. The third sample was treated with drug alone under the same dose. The H-VNBs mode (sample 1) showed 3-fold higher parasiticidal efficacy than chloroquine alone (sample 3) and rapidly reduced the level of iRBCs to $5-7 \%$ of that in the untreated sample in 24 hours (Figure 5B). The concentration of uninfected RBCs did not show any detectable changes 24 or 48 hours after the H-VNB treatment. The maximal parasiticidal effect was observed in the sample 2 with a combination of $\mathrm{H}-\mathrm{VNB}$ and drug treatment (Figure 5B). However, clearly the major parasiticidal effect was provided by the H-VNBs.

The untreated iRBCs had a level of infection of 6.3 and $16.4 \%$ at 24 and 48 hours (Figure 5A). In contrast, the H-VNB-treated samples demonstrated rapid reduction in the infection level to almost zero (0.2 and $0.5 \%$ at 24 and 48 hours, respectively). This experiment demonstrates rapid, irreversible parasiticidal effect of $\mathrm{H}-\mathrm{VNBs}$ and its advantage over the standard anti-malaria drug. The hemozoin-generated vapor nanobubbles destroy parasites several times more efficiently and much more rapidly than $1 \mu \mathrm{M}$ chloroquine and would still be effective in killing drug resistant malaria parasites.

\section{Influence of the laser pulse parameters on the parasiticidal efficacy of $\mathrm{H}-\mathrm{VNBs}$}

To analyze the influence of the laser parameters on the generation of $\mathrm{H}-\mathrm{VNBs}$ and their parasiticidal effect, we compared the effect of 70 ps and 14 ns pulses at variable levels of laser fluence. The duration (lifetime) of each H-VNB optical scattering trace was measured for hemozoin crystals in water and in iRBCs as function of a laser pulse fluence and duration (Figure 6A\&B). For both hemozoin in water and human iRBCs, we observed that the threshold laser fluence for H-VNB generation and the lifetime of the resulting vapor bubble depend upon laser pulse duration. Under identical laser fluence, the shorter 70 ps 
pulse generates the largest $\mathrm{H}-\mathrm{VNBs}$ and requires the minimal threshold fluence (about $10 \mathrm{~mJ} / \mathrm{cm}^{2}$ ), whereas the longer $14 \mathrm{~ns}$ pulse has a higher $\mathrm{H}-\mathrm{VNB}$ generation threshold (about $40 \mathrm{~mJ} / \mathrm{cm}^{2}$ ) and generates smaller H-VNBs, as measured by lifetimes (Figure 6A\&B). This pulse duration effect is determined by the size of the optical absorber. Hemozoin nanocrystals are 50-1000 $\mathrm{nm}$ in size [13-15] and generate $\mathrm{H}-\mathrm{VNBs}$ more efficiently with a 70 ps pulse rather than with the much longer 14 ns pulse (Figure $6 \mathrm{~A} \& \mathrm{~B})$. The longer pulse allows heat losses due to thermal diffusion from hemozoin nanocrystal while excitation is still occurring. Absorbance of the $70 \mathrm{ps}$ picosecond pulse by the hemozoin nanocrystal results in a thermal confinement and rapid evaporation of the surrounding water layers, resulting in higher efficacy of $\mathrm{H}-\mathrm{VNB}$ generation.

For the destruction of the parasites, increasing the fluence of the short $70 \mathrm{ps}$ pulse beyond $40 \mathrm{~mJ} / \mathrm{cm}^{2}$ does not enhance the parasiticidal efficacy (Figure 6C), and a longer 14 ns pulse shows much lower parasiticidal efficacy (Figure 6C). At the same time, a longer, 14 ns pulse treatment lysed roughly $25 \%$ of the uninfected RBCs, due to more delocalized photothermal heating. In contrast, a shorter 70 ps pulse treatment lysed less than $2 \%$ of uninfected RBCs Thus, a short picosecond laser pulse provides both better efficacy for H-VNB generation and much higher parasiticidal selectivity (resulting in much higher safety for normal blood cells). The parasiticidal effect of $\mathrm{H}-\mathrm{VNB}$ is determined by the mechanical disruptive impact of the expanding and collapsing vapor nanobubble, which is the process that creates locally high pressure gradients [27-29]. The mechanical impact and therapeutic strength of the vapor nanobubble increase with its maximal size [30, 31]. Consequently, we measured the maximal size of $\mathrm{H}-\mathrm{VNBs}$ as a function of the parasite development stage using a fixed level of fluence for the shorter, 70 ps laser pulse (Figure 6D). The development of a parasite from early ring to the mature schizont stage caused multi-fold increases in the H-VNB lifetime, which is a metric of its maximal size. As shown, the parasiticidal strength of the H-VNB is amplified during the development and maturation of the parasite from the ring to schizont stage because this process also leads to an increase in the size of hemozoin nanocrystals [13-15]. We have studied this effect of size of the optically absorbing nanoparticle on the lifetime of the laser pulse-induced vapor nanobubble more extensively using gold nanospheres [17, 31, 32] and the current results for hemozoin nanocrystals fall in line with these previous observations.
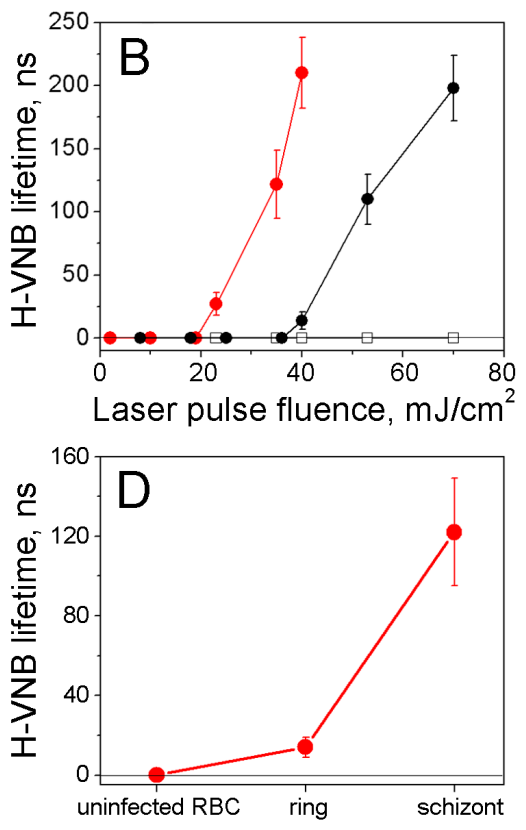

Parasite stage

Figure 6. Parameters for H-VNB generation measured by light scattering traces. (A) Dependence of the H-VNB lifetime (a metric for maximal size) upon the single laser pulse fluence and duration for hemozoin crystals in water: red circles - $532 \mathrm{~nm}, 70 \mathrm{ps}$; black circles - $532 \mathrm{~nm}$, $14 \mathrm{~ns}$. (B) Dependence of the $\mathrm{H}-\mathrm{VNB}$ lifetime on laser pulse fluence and duration for iRBCs with mature schizont stages of parasites and uninfected RBCs: red circles - iRBC, laser pulse at $532 \mathrm{~nm}, 70 \mathrm{ps}$; solid black circles - iRBC, laser pulse at $532 \mathrm{~nm}, 14 \mathrm{~ns}$; hollow black squares - normal RBC, laser pulse at $532 \mathrm{~nm}, 70 \mathrm{ps}$. (C) Levels of infected cells $24 \mathrm{~h}$ and $48 \mathrm{~h}$ after laser flow treatment: magenta: iRBCs treated with laser pulse of $14 \mathrm{~ns}, 70 \mathrm{~mJ} / \mathrm{cm}^{2}$; green: iRBCs treated with laser pulse of 70 ps, $30 \mathrm{~mJ} / \mathrm{cm}^{2}$; green stripes: iRBCs treated with laser pulse of $70 \mathrm{ps}, 130 \mathrm{~mJ} / \mathrm{cm}^{2}$. (D) Dependence of the H-VNB lifetime for individual iRBC upon the malaria parasite stage under a single laser pulse excitation $\left(532 \mathrm{~nm}, 70 \mathrm{ps}, 35 \mathrm{~mJ} / \mathrm{cm}^{2}\right)$. 


\section{Discussion}

Laser pulse-induced photothermal destruction of iRBCs was attempted shortly after the invention of the laser [33]. The original approach used iRBCs that were pre-treated with an absorbing dye, a much longer pulse, and 1000-fold higher energy than our current method to kill the parasites. However, the selectivity for iRBC destruction was low and many surrounding normal cells were damaged due presumably to non-specific heating of cytoplasmic hemoglobin. Magnetic heating of hemozoin was also proposed as method for selectively killing malaria parasites [34] but significant thermal diffusive losses due to long excitation times [19] markedly reduced the efficacy and selectivity of this method.

In contrast, our short, low energy laser pulses result in highly localized mechanical impacts by generating $\mathrm{H}-\mathrm{VNBs}$ around the intra-parasital hemozoin particles without heating or damaging uninfected cells. The nature of H-VNB-based destruction of the malaria parasite is effectively mechanical, not thermal, because the rapidly formed vapor nanobubble thermally insulates the outer water layer from the super-heated particle [17]. The intra-parasital nature of the H-VNB is provided by the location of hemozoin in the pathogen's food vacuole. The explosive mechanical impact of the transient nanobubble is destructive to living matter within its maximal radius (quantified through its lifetime in our studies). The fact that the vapor nanobubble also appears to destroy the host cell means that the red blood cell cytoplasm is also exposed an intense pressure gradient. The speed of parasite destruction is determined by the duration of the mechanical impact, i.e. by the H-VNB lifetime, and can be estimated to occur in a few hundred nanoseconds up to 1 microsecond. Thus, compared to current treatments, which take hours to days, the $\mathrm{H}-\mathrm{VNB}$ mechanism provides instantaneous destruction of the parasite. Although the spatial and temporal scales of this process do not allow direct optical imaging of bubble development with nanometer resolution, the spatial coincidence of the nanobubble, parasite DNA, and destruction of the host RBC in single cell experiments (Figure 2), and the dramatic decrease in parasite level after bulk treatment (Figure 5) show unambiguously that a single $\mathrm{H}-\mathrm{VNB}$ can completely destroy the malaria parasite. The data in Figure 5 also demonstrate that no release of viable parasites into blood occurs after the H-VNB treatment and concomitant iRBC lysis.

One limitation of hemozoin-induced nanobubbles involves the in vivo accessibility of parasites to photoexcitation in the liver or in iRBCs adherent to blood vessel walls. In principle, $\mathrm{H}-\mathrm{VNB}$ theranostics can be applied for elimination of parasites from blood, using a transdermal; pulsed laser system similar to the diagnostic system proposed in [16] but would require a very long treatment time. Whole blood could also be treated extracorporeally using standard clinical equipment such as an extracorporeal membrane oxygenation or a dialysis apparatus and directing the laser pulses through a short transparent cuvette (Figure 3). In our bulk treatment model the experimental flow rate was limited by the energy of the laser pulse and by the pulse repetition rate. Commercial lasers with 200-400 mJ/pulses and 100 $\mathrm{Hz}$ repetition rates would allow an increase in the treatment rate to $500 \mathrm{ml} / \mathrm{min}$. This rate would allow the extracorporeal treatment of all the blood cells of a patient in 10 to 20 minutes. In addition, replacing the green visible wavelength (as employed in this work) by near-infrared laser pulses at $\mathrm{H}$-VNB-specific wavelength of $672 \mathrm{~nm}$ [16] would significantly reduce the probability of photodamage to normal blood cells because optical absorbance at $672 \mathrm{~nm}$ is negligible for all endogenous blood components. However, at present, the most realistic application of the described technology is application to the screening and treatment of donor blood from a blood bank.

The theranostic use of H-VNBs can be applied by the simultaneous detection and destruction of the malaria parasite. In vitro detection of malaria in donated blood would employ the optical scattering effect of H-VNB (Figure 2C\&D). In a flow system, such detection can be realized with the broad continuous probe laser beam of low power (Figure 3) that illuminates the volume exposed to the laser pulse, and with an optical detector that registers scattering of the probe laser light by H-VNBs. The H-VNB-specific signal of the detector (Figure $2 \mathrm{C}$ or $\mathrm{D}$ ) will report the presence of malaria infection and at the same time will result in real time the destruction of the malarial parasite in which the H-VNB is generated. This method is based on previous well-established optical scattering methods for the detection of laser-induced vapor nanobubbles in cells containing exogenous nanoparticles $[27,28]$, including the monitoring of the mechanical damage of cells $[35,36]$ and theranostic application of intracellular vapor nanobubbles [30, 36, 37].

Further understanding of the diagnostic and parasiticidal mechanisms of nanobubbles for malaria infections requires additional research to determine their specific application. Hemozoin- and laser pulse-induced vapor nanobubbles will detect and destroy any stage (including gametocytes) and any type of malaria parasite that contains hemozoin nanocrystals. Thus the H-VNB technology has the potential to eliminate drug-resistant strains and offers 
a totally new cell theranostic approach where the detection and destruction of the pathogen are united in one rapid drug-free procedure, in which the efficacy is determined by the size of hemozoin nanocrystals in the parasites.

\section{Acknowledgements}

Authors thank Drs. Janet Braam and John Olson of Rice University for their generous support of earlier portions our malaria project and for help in editing of the current paper, Dr. D.J. Sullivan of the Johns Hopkins Malaria Research Institute for consultations on malaria research and Dr. R. Ware of Rice University for providing human blood samples. This work was partially supported by National Institute of Health Grants R01GM094816 (DOL) and S10RR026399-01 (Confocal microscope).

\section{Competing Interests}

The authors have declared that no competing interest exists.

\section{References}

1. The malERA consultative group on monitoring, evaluation, and surveillance. A research agenda for malaria eradication: diagnoses and diagnostics. PLoS Med 2011; 8: e1000396.

2. [Internet] WHO. "World Malaria Report: 2010," in WHO Library Cataloguing in Publication Data. http://www.who.int/malaria/world_ malaria_report_2010/en/index.html.

3. Kappe SHI, Vaughan AM, Boddey JA, Cowman AF. That was then but this is now: malaria research in the time of an eradication agenda. Science 2010; 328: 862-6.

4. Wellems TE. Plasmodium chloroquine resistance and the search for a replacement antimalarial drug. Science 2002; 298: 124-6.

5. Baird JK. Effectiveness of antimalarial drugs. N Engl J Med 2005; 352: 1565-77.

6. Gryseels C, Uk S, Erhart A, Gerrets R, Sluydts V, Durnez L, Muela Ribera J, Hausmann Muela S, Menard D, Heng S, Sochantha T, D'Alessandro U, Coosemans M, Peeters Grietens K. Injections, cocktails and diviners: therapeutic flexibility in the context of malaria elimination and drug resistance in Northeast Cambodia. PLos One 2013; 8: E80343.

7. Talisuna AO, Karema C, Ogutu B, Juma E, Logedi J, Nyandigisi A, Mulenga M, Mbacham WF, Roper C, Guerin PJ, D'Alessandro U, Snow RW. Mitigating the threat of artemisinin resistance in Africa: improvement of drug-resistance surveillance and response systems. Lancer Infect Dis 2012; 12:8888-96

8. Seder RA, Chang LJ, Enama ME, Zephir KL, Sarwar UN, Gordon IJ, Holman LA, James ER, Billingsley PF, Gunasekera A, Richman A, Chakravarty S, Manoj A, Velmurugan S, Li M, Ruben AJ, Li T, Eappen AG, Stafford RE, Plummer SH, Hendel CS, Novik L, Costner PJ, Mendoza FH, Saunders JG, Nason MC, Richardson JH, Murphy J, Davidson SA, Richie TL, Sedegah M, Sutamihardja A, Fahle GA, Lyke KE, Laurens MB, Roederer M, Tewari K, Epstein JE, Sim BK, Ledgerwood JE, Graham BS, Hoffman SL; VRC 312 Study Team. Protection against malaria by intravenous immunization with a nonreplicating sporozoite vaccine. Science 2013; 341: 1359-65.

9. Roestenberg M, Bijker EM, Sim BK, Billingsley PF, James ER, Bastiaens GJ, Teirlinck AC, Scholzen A, Teelen K, Arens T, van der Ven AJ, Gunasekera A, Chakravarty S, Velmurugan S, Hermsen CC, Sauerwein RW, Hoffman SL. Controlled human malaria infections by intradermal injection of cryopreserved Plasmodium falciparum sporozoites. Am J Trop med Hyg 2013; 88: 5-13.

10. Sullivan DJ, Gluzman IY, Goldberg DE. Plasmodium hemozoin formation mediated by histidine-rich proteins. Science 1996; 271: 219-22.

11. Lee JY, Clarke ML, Tokumasu F, Lesoine JF, Allen DW, Chang R, Litorja M, Hwang J. Absorption-based hyperspectral imaging and analysis of single erythrocytes. IEEE J Sel Top Quantum Electron 2012; 18: 1130-9.

12. Gligorijevic B, Purdy K, Elliott DA, Cooper RA, Roepe PD. Stage independent chloroquine resistance and chloroquine toxicity revealed via spinning disk confocal microscopy. Mol Biochem Parasitol 2008; 159: 7-23.

13. Egan TJ. Recent advances in understanding the mechanism of hemozoin (malaria pigment) formation. J Inorg Biochem 2008; 102: 1288-99.

14. Noland GS, Briones N, Sullivan DJ Jr. The shape and size of hemozoin crystals distinguishes diverse Plasmodium species. Mol Biochem Parasitol 2003; 130: 91-9.

15. Coban C, Yagi M, Ohata K, Igari $Y$, Tsukui T, Horii T, Ishii KJ, Akira S. The malarial metabolite hemozoin and its potential use as a vaccine adjuvant. Allergol Int 2010; 59: 115-24.

16. Lukianova-Hleb EY, Campbell KM, Constantinou PE, Braam J, Olson JS, Ware RE, Sullivan DJ, Lapotko DO. Hemozoin-generated vapor nanobubbles for transdermal reagent and needle-free detection of malaria. Proc Natl Acad Sci U S A 2014; 111: 900-5.

17. Lukianova-Hleb Y, Hu Y, Latterini L, Tarpani L, Lee S, Drezek R, Hafner $\mathrm{JH}$, Lapotko DO. Plasmonic nanobubbles as transient vapor nanobubbles generated around plasmonic nanoparticles. ACS Nano 2010; 4: 2109-23.

18. Brinkmann R, Neumann J. Self-limited growth of laser-induced vapor bubbles around single microabsorbers. Appl Phys Lett 2008; 93: 033901.

19. Anderson R, Parrish J. Selective photothermolysis: precise microsurgery by selective absorption of pulsed radiation. Science 1983; 220: 524-7.

20. Lukianova-Hleb EY, Sassaroli E, Jones A, Lapotko D. Transient photothermal spectra of plasmonic nanobubbles. Langmuir 2012; 28: 4858-66.

21. Lukianova-Hleb EY, Ren X, Zasadzinski JA, Wu X, Lapotko D. Plasmonic nanobubbles enhance efficacy and selectivity of chemotherapy against drug-resistant cancer cells. Adv Mater 2012; 24: 3831-7.

22. Lukianova-Hleb EY, Lapotko DO. Experimental techniques for imaging and measuring transient vapor nanobubbles. Appl Phys Lett 2012; 101: 264102.

23. Trager $W$, Jensen J. Human malaria parasites in continuous culture. Science 1976; 193: 673-5.

24. Cho S, Kim S, Kim Y, Park YK. Optical imaging techniques for the study of malaria. Trends Biothechnol 2012; 30: 71-9.

25. Guy R, Liu P, Pennefather P, Crandall I. The use of fluorescence enhancement to improve the microscopic diagnosis of falciparum malaria. Malar J 2007; 6: 89.

26. Sutanto I, Endawati D, Ling LH, Laihad F, Setiabudy R, Baird JK. Evaluation of chloroquine therapy for vivax and falciparum malaria in southern Sumatra, Western Indonesia. Malar J 2010; 9: 52.

27. Lin CP, Kelly MW, Sibayan SAB, Latina MA, Anderson RR. Selective cell killing by microparticle absorption of pulsed laser radiation. IEEE J Quantum Electron 1999; 5: 963-8.

28. Kelly MW. Intracellular cavitation as a mechanism of short-pulse laser injury of the retinal pigment epithelium. Medford, MA: PhD Dissertation, Tufts Univ. 1997.

29. Lukianova-Hleb EY, Oginsky AO, Samaniego AP, Shenefelt DL, Wagner DS, Hafner JH, Farach-Carson MC, Lapotko DO. Tunable plasmonic nanoprobes for theranostics of prostate cancer. Theranostics 2011;1:3-17.

30. Lukianova-Hleb EY, Hanna EY, Hafner JH, Lapotko DO. Tunable plasmonic nanobubbles for cell theranostics. Nanotechnology 2010; 21: 085102.

31. Lukianova-Hleb EY, Ren X, Sawant RR, Wu X, Torchilin VP, Lapotko DO. On-demand cancer cell-specific plasmonic nanobubble-mediated enhancement of intracellular drug release and radiation effects. Nat Medicine 2014; DOI: 10.1038/nm.3484.

32. Lapotko D. Optical excitation and detection of vapor bubbles around plasmonic nanoparticles. Opt Express 2009; 17: 2538-56.

33. Rounds D, Opel W, Olson RS, Sherman IW. The potential use of laser energy in the management of malaria. Biochem Biophys Res Commun 1968; 32: 616-23.

34. Hegg M, Horning MP, et al. Systems, devices, and methods including paramagnetic oscillation, rotation and translation of hemozoin asymmetric nanoparticles in response to multi-harmonic optical detection of the presence of hemozoin, US Application Number 12/658617. 2010.

35. Lapotko D, Shnip A, Lukianova E. Photothermal detection of laser-induced damage in single intact cells. Lasers Surg Med 2003; 33: 320-329.

36. Lapotko D, Lukianova E, Shnip A, Zheltov G, Potapnev M, Oraevsky A, Savitskiy V, Klimovich O. Photothermal microscopy and laser ablation of leukemia cells targeted with gold nanoparticles. Proc SPIE 2005; 5697: 82-89.

37. Lapotko D. Laser-induced bubbles in living cells. Lasers Surg Med 2006; 38: $240-248$. 